\title{
Peripheral Ischemia, CTCAE
}

National Cancer Institute

\section{Source}

National Cancer Institute. Peripheral Ischemia, CT CAE. NCI Thesaurus. Code C143749.

A disorder characterized by impaired circulation to an extremity. 